\title{
Reduced Growth Cone Motility in Cultured Neurons from Drosophila Memory Mutants with a Defective cAMP Cascade
}

\author{
Yun-Taik Kim and Chun-Fang Wu \\ Department of Biological Sciences, University of lowa, lowa City, lowa 52242
}

\begin{abstract}
Drosophila memory mutants dunce (dnc) and rutabaga (rut) are known to have altered intracellular cAMP levels, nerve terminal growth, and plasticity of synaptic transmission. Because the growth cone is responsible for neurite outgrowth and synaptogenesis, video microscopy was used to examine growth cone morphology and behavior of mutant neurons in larval CNS cultures. We found that growth cone exploratory movement was nearly arrested by both mutations, even though they change cAMP levels in opposite directions. The dnc phenotype could be mimicked by normal neurons when perfused with dibutyryl cAMP (db-cAMP) or forskolin. In contrast, rut growth
\end{abstract}

cones became active when perfused with db-cAMP. Furthermore, motility was also restored by counterbalancing the effects of the two genes in double mutants, indicating that dynamic control of growth cone motility in developing Drosophila neurons requires optimal cAMP levels within an operational range. These findings represent the first demonstration of altered growth cone properties in learning and memory mutants and establish in a natural setting the role of CAMP in growth cone motility and neuronal plasticity.

Key words: dnc; rut; growth cone; lamellipodia; neuronal plasticity; learning; memory; cAMP; Drosophila; CNS; culture
The Drosophila mutants $d n c$ and rut were isolated because of their learning deficiencies (Dudai et al., 1976; Aceves-Pina et al., 1983; Livingstone et al., 1984). Biochemical assays of embryos, larvae, and adults indicate that $d n c$ mutations affect cAMP phosphodiesterase (cAMP PDE), leading to increased intracellular cAMP (Byers et al., 1981; Davis and Kiger, 1981), and rut mutations affect $\mathrm{Ca}^{2+} /$ calmodulin-sensitive adenylyl cyclase (AC), lowering $\mathrm{Ca}^{2+}$-dependent cAMP synthesis (Dudai et al., 1983; Livingstone et al., 1984). Indeed, molecular cloning and DNA sequence analysis confirmed that $d n c$ codes for the enzyme cAMP PDE (Chen et al., 1986; Qiu et al., 1991) and rut codes for a form of AC (Levin et al., 1992). Morphological and physiological alterations have also been reported in the $d n c$ and rut nervous systems. The number of nerve fibers in the mushroom bodies, which are involved in olfactory learning, is regulated in an experiencedependent manner (Technau, 1984; Heisenberg et al., 1985). Neither $d n c$ nor rut flies displayed such developmental plasticity (Balling et al., 1987; Heisenberg et al., 1995). Sensory fatigue and adaptation in $d n c$ and rut adult thoracic bristles are altered, and morphological abnormalities have been found in the same sensory axon arbors (Corfas and Dudai, 1990, 1991). Furthermore, the numbers of axonal terminal branches and varicosities of larval motor neurons are increased on identified muscles in different $d n c$ alleles, an effect that could be suppressed by rut in double-mutant combinations (Zhong et al., 1992). At the same neuromuscular junctions, activity-dependent plasticity in transmitter release, i.e.,

Received March 19, 1996; revised June 25, 1996; accepted June 26, 1996.

This work was supported by National Institutes of Health grants to C.-F.W. and by a KOSEF-Korea Grant to Y.-T.K. We thank Mr. Peter Taft for technical assistance in data collection for Figures 5-8, and Dr. Jeff E. Engel for comments on this manuscript. Some of the image processing was performed in the Cell Motility Core Facilities in the Department of Biological Sciences, University of Iowa.

Correspondence should be addressed to Dr. Chun-Fang Wu, Department of Biological Sciences, University of Iowa, Iowa City, IA 52242.

Dr. Kim's present address: Department of Life Science, Sogang University, One Sinsu Dong, Seoul 121-742, Korea.

Copyright (C) 1996 Society for Neuroscience $0270-6474 / 96 / 165593-10 \$ 05.00 / 0$ facilitation and post-tetanic potentiation, is severely reduced in different $d n c$ and rut alleles (Zhong and Wu, 1991).

The above phenotypic abnormalities illustrate the close relationship between developmental and physiological plasticity. Because the cellular mechanisms regulating neurite branching and synaptic contacts in Drosophila have not been well established, analysis of the neuronal growth process in these mutants may provide insight into the role of cAMP in developmental plasticity. We examined cultured $d n c$ and rut neurons dissociated from the larval CNS. This culture system is suitable for pharmacological studies on isolated neurons (Wu et al., 1983; Wu, 1988) and enables direct monitoring of growth cone behavior under controlled conditions (Kim and Wu, 1987, 1991a).

Preliminary reports of part of this work have been published previously (Kim and Wu, 1991b; Kim et al., 1995).

\section{MATERIALS AND METHODS}

Animal stocks. The wild-type strain Canton-Special (CS), three different alleles of the $d n c$ locus, $d n c^{1}, d n c^{M 11}, d n c^{M 14}$, one allele of the rut gene, $r u t^{1}$, double mutants $d n c^{1} r u t^{1}$ and $d n c^{M 14} r^{1} t^{1}$, and $r u t^{1} / D f(1) K A 9$ of Drosophila melanogaster were raised on standard Drosophila medium at $22^{\circ} \mathrm{C}$. $K A 9$ carries a small $\mathrm{X}$-chromosome deletion uncovering rut. Male $d n c^{M 11}$ and $d n c^{M 14}$ were used in the experiments and maintained in a balanced stock with attached X-chromosomes because of their female sterility (Mohler, 1977).

Dissociated larval CNS cell culture. Dissociated neurons from the wildtype strain $C S$ were used for normal controls throughout the experiments. The procedure for culturing neurons from Drosophila larvae was identical to that reported previously (Kim and Wu, 1991a). Briefly, third instar larvae of the wondering stage were collected and dissected in culture medium after being surface-sterilized in $70 \%$ ethanol. Their brain and ganglion complexes were cut and incubated in $\mathrm{Ca}^{2+}$ - and $\mathrm{Mg}^{2+}$-free saline solution containing $0.5 \mathrm{mg} / \mathrm{ml}$ collagenase (Type I, Sigma, St. Louis, MO). After dissociation of tissue fragments in culture medium, the cell suspension was plated on uncoated glass coverslips. Growth cones of wild-type neurons show more active movement on uncoated glass coverslips than on polylysine-coated ones (Kim and Wu, 1987, 1991a), as in the cases of neuronal cultures of vertebrate species (Bray and Chapman, 1985). Therefore, uncoated coverslips were used in this study to accentuate differences between normal and mutant growth cones. Culture 


\begin{tabular}{|c|c|c|c|c|c|c|}
\hline \multirow[b]{2}{*}{ Genotype } & \multirow{2}{*}{$\begin{array}{l}\text { Number of } \\
\text { cells (cultures) } \\
\text { examined }\end{array}$} & \multirow{2}{*}{$\begin{array}{l}\text { Cells with } \\
\text { growth } \\
\text { cone }\end{array}$} & \multicolumn{4}{|c|}{ Growth cone phase quality vs activity } \\
\hline & & & Light & (act/inact) & Dark & (act/inact) \\
\hline Normal & $183(8)$ & $33.3 \%$ & $60.7 \%$ & $(23 / 14)^{*}$ & $39.3 \%$ & $(0 / 24)$ \\
\hline$d n c^{1}$ & $214(8)$ & $33.6 \%$ & $63.9 \%$ & $(0 / 31)$ & $36.1 \%$ & $(0 / 14)$ \\
\hline$r t^{l}$ & $193(7)$ & $31.6 \%$ & $62.3 \%$ & $(0 / 38)$ & $37.7 \%$ & $(0 / 23)$ \\
\hline$d n c^{l} r u t^{l}$ & $52(4)$ & $48.1 \%$ & $80.0 \%$ & $(15 / 5)^{*}$ & $20.0 \%$ & $(0 / 5)$ \\
\hline
\end{tabular}

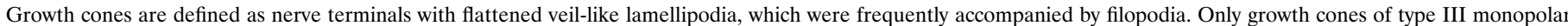

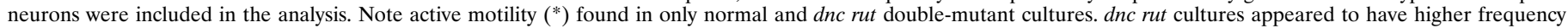
of active, phase-light growth cones. act, Active; inact, inactive.

medium consisted of $90 \%$ revised Schneider's Drosophila medium (Life Technologies, Gaithersburg, MD) and $10 \%$ heat-inactivated fetal bovine serum containing $50 \mathrm{U} / \mathrm{ml}$ penicillin and $50 \mu \mathrm{g} / \mathrm{ml}$ streptomycin sulfate (Wu et al., 1983).

Video microscopy. Within $2 \mathrm{~d}$ of culture, growth cones with flat veil-like lamellipodia in type III neurons (Wu et al., 1983; Kim and Wu, 1991a) were selected for observation. Contrast-enhanced video microscopy was performed by using a Zeiss IM35 inverted microscope and a Hamamatsu C-2400 video camera with a newvicon tube in conjunction with a contrast enhancement circuit, as described previously (Kim and Wu, 1991a). Continuous video recording for individual growth cones was made in real time for 3-12 min (sometimes for up to $30 \mathrm{~min}$ ).

Pharmacology. Dibutyryl cAMP (db-cAMP) (Sigma) was dissolved in Drosophila normal saline (Wu et al., 1983) to make the stock solution $(500 \mu \mathrm{M})$. Forskolin (Calbiochem, La Jolla, CA) stock solution $(50 \mathrm{mM})$ was prepared in dimethyl sulfoxide (DMSO), and in perfusion medium the final DMSO concentration was $0.1 \%$. Control experiments showed that $0.1 \%$ DMSO had no apparent effects on growth cone morphology and motility. For perfusion experiments, coverslips with cultures were mounted on a double-coverslip chamber (Forscher et al., 1987) in a configuration with two open ends suitable for perfusion. Cells were first perfused with normal medium before exposure to test solutions; continuous perfusion at a fixed flow rate $(150-200 \mu \mathrm{l} / \mathrm{min})$ was maintained at all times. The dead volume in the perfusion system delayed the arrival of the perfusate at the neuronal culture for $\sim 30 \mathrm{sec}$.

Analysis of growth cone morphology and behavior. The method to analyze growth cone morphology and motility was essentially the same as reported previously (Soll et al., 1988; Kim and Wu, 1991a), with an improved version of computer analysis software (DIAS, cf. Soll, 1995). Time-lapse sequences of growth cone images were analyzed for their motility by a combination of photographic and graphic procedures. Lamellipodial images were traced from photographs and subjected to computer-assisted analysis. The methods for producing difference pictures and motility indices of lamellipodia, i.e., (area of expansion + area of retraction)/(area of earlier image + area of later image), and the formula for calculating roundness, i.e., $\left(4 \pi\right.$ area/perimeter $\left.{ }^{2}\right)$, have been described previously (Soll et al., 1988; Kim and Wu, 1991a; Soll, 1995).

\section{RESULTS}

\section{Abnormal growth cone motility in dnc and rut neurons}

It is important to examine multiple $d n c$ and rut alleles so that any abnormalities observed could be attributed to the mutational effects of these genes. We studied several independent isolates of the two genes, including $d n c^{1}, d n c^{M 11}, d n c^{M 14}, r u t^{1}$, and $r u t^{1}$, $D f(1) K A 9$. (KA9 is a deletion uncovering rut.)

The larval CNS culture consists of several types of neurons at different stages of differentiation (Wu et al., 1983). Our study focused on monopolar type III neurons because they have larger cell bodies and display more conspicuous growth cones in which morphometric and motility parameters have been studied in detail (Kim and Wu, 1991a). Not all type III neurons at a given time showed growth cones with lamellipodia. Only growth cones equipped with lamellipodia in isolated neurons, but not in neuronal clusters, were included in this study.

In general, no obvious differences in growth cone morphology (Fig. 1) and frequency of occurrence (Table 1) were found between normal and mutant cultures. (There seems to be a small increase in the frequency of occurrence of $d n c^{1} r u t^{1}$ neurons with lamellipodial growth cones, but the smaller sample size for this genotype shown in Table 1 precludes a firm conclusion.) In a
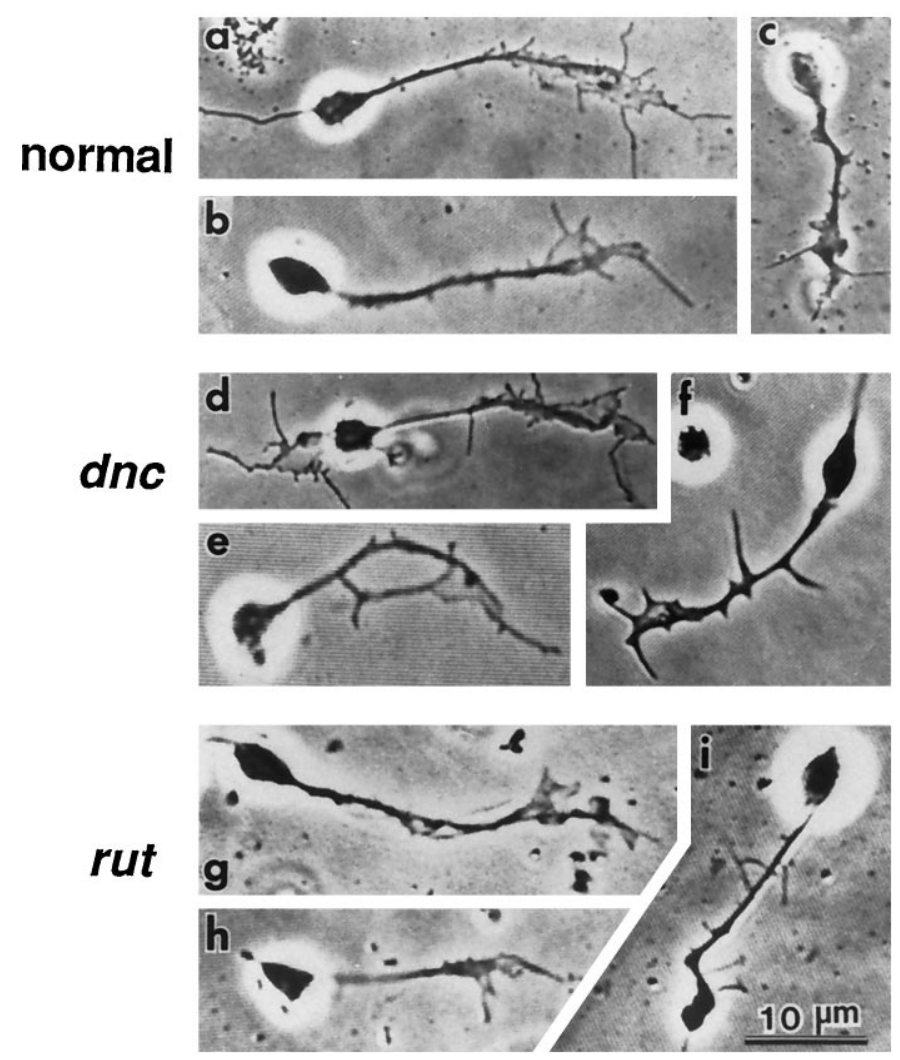

Figure 1. Neurons with growth cones in cultures from normal, $d n c^{1}$, and $r^{\prime} t^{1}$ larval CNS. Approximately $60-65 \%$ of growth cones in both mutant and normal cultures showed lighter images $(a, b, d, e, g, h)$ in phasecontrast optics. The remaining growth cones appeared thicker and darker $(c, f, i)$. The morphology of phase-light growth cones of $d n c^{l}$ and $r u t^{l}$ neurons was indistinguishable from that of normal neurons. The phasedark growth cones of both $d n c^{1}$ and $r u t^{1}$ neurons appeared darker than those in normal neurons. Cultured neurons from the wild-type strain $C S$ were used as normal controls in this and the following figures (see Materials and Methods). 
Table 2. Growth cone morphology and motility of normal and mutant neurons in culture

Growth cone morphology

\begin{tabular}{|c|c|c|c|c|c|c|c|}
\hline \multirow[b]{2}{*}{ Genotype } & \multicolumn{2}{|l|}{ Filopodia } & \multicolumn{2}{|l|}{ Lamellipodia } & \multirow[b]{2}{*}{$n$} & \multicolumn{2}{|c|}{ Growth cone motility } \\
\hline & No/GC $\pm \mathrm{SEM}$ & Length \pm SEM & Area \pm SEM & Roundness \pm SEM & & Index $\pm \mathrm{SD}$ & $n$ \\
\hline Normal & $3.1 \pm 0.2$ & $5.0 \pm 0.3$ & $18.3 \pm 1.0$ & $0.38 \pm 0.02$ & 53 & $0.36 \pm 0.09$ & 5 \\
\hline$d n c^{M 11}$ & $3.5 \pm 0.3$ & $5.2 \pm 0.4$ & $16.2 \pm 2.5$ & $0.37 \pm 0.02$ & 39 & $0.09 \pm 0.03^{*}$ & 4 \\
\hline$d n c^{M 14}$ & $3.3 \pm 0.7$ & $4.6 \pm 0.5$ & $15.5 \pm 1.8$ & $0.29 \pm 0.09$ & 10 & $0.07 \pm 0.03^{*}$ & 4 \\
\hline$r u t^{l}$ & $2.9 \pm 0.2$ & $5.2 \pm 0.4$ & $13.4 \pm 0.9$ & $0.41 \pm 0.02$ & 60 & $0.07 \pm 0.03^{*}$ & 4 \\
\hline
\end{tabular}

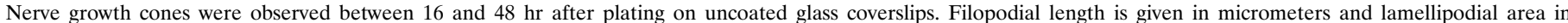

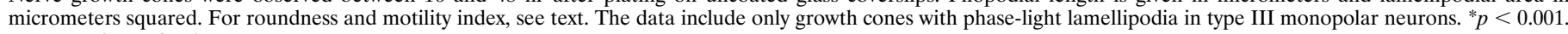
ND, Not determined.

series of systematic surveys designed for random sampling in 16to 48 -hr-old cultures, $33.3 \%$ of type III monopolar neurons in normal cultures displayed extended lamellipodia at the tip of the neurite, whereas $d n c^{1}, r u t^{1}$, and $r u t^{1} / D f(1) K A 9$ cultures showed $33.6 \%, 31.6 \%$, and $34.3 \%$, respectively (Table 1). A population of these lamellipodia appeared lighter and thinner in phase optics $\left(60.7 \%, 63.9 \%, 62.3 \%\right.$, and $64.7 \%$ in normal, $d n c^{1}, r u t^{1}$, and $r u t^{1} / D f(1) K A 9$, respectively). The rest of the growth cones appeared darker and thicker and were nearly completely immobile (Fig. 1c,f,i). As reported previously, these morphological features are associated with more advanced stages of development (Hadley et al., 1985; Kim and $\mathrm{Wu}, 1991 \mathrm{a}$ ) and are more abundant in cultures $>3 \mathrm{~d}$ old. These well differentiated neurons were excluded from further morphometric and motility studies described below. The immobile phase-dark growth cones appeared even darker in the mutant cultures (Fig. 1f,i). Table 2 summarizes several morphometric parameters, including the number and length of filopodia associated with the lamellipodium, and the area and roundness of the lamellipodium, determined in 16- to 48-hr-old cultures. Roundness here indicates degree of convexity and concavity of the boundary of the lamellipodium (filopodia excluded; see Kim and $\mathrm{Wu}, 1991 \mathrm{a})$. In general, mutant growth cones did not show distinct differences. The only small differences were the reduction in the area of lamellipodia in $r u t^{1}$ and the roundness of lamellipodia in $d n c^{M 14}$.

Despite the similarity in morphology of the phase-light growth cones, striking differences in the motility of growth cone lamellipodia were evident when normal and mutant neurons were compared (Fig. 2; compare Fig. 8). In normal cultures, $\sim 62 \%(23 / 37$ in the above survey; see Table 1) of lamellipodia showed high motility, readily detectable at 5 sec intervals. Ruffle movement of lamellipodia and filopodial extension and retraction from their edges were observed at each $5 \mathrm{sec}$ interval in normal cultures (Fig. 2 , top). In contrast, growth cones of $d n c$ alleles and rut neurons were relatively stable, showing greatly reduced motility (Fig. 2 for $d n c^{1}, d n c^{M 11}$, and $r u t^{1}$; compare Fig. 8 for $d n c^{M 14}$ and $\left.r u t^{1} / K A 9\right)$. Lamellipodial ruffling was not detected in $d n c$ and rut growth cones during several $5 \mathrm{sec}$ intervals. Only minor changes of lamellipodial shape could be detected after longer intervals of quiescence.

This can be visualized better with difference pictures (Kim and $\mathrm{Wu}, 1991 \mathrm{a})$, which show areas of change attributable to lamellipodial expansion and retraction. In Figure 3, areas in black were obtained by superimposing two consecutive frames to show the sequence of motility of a growth cone at $5 \mathrm{sec}$ intervals. It is clear that the change in area of normal growth cones was greater than that of $d n c^{1}$ and $r u t^{1}$ growth cones.

The dynamic changes of growth cones were quantified further by using a motility index. The area changes in difference pictures at $5 \mathrm{sec}$ intervals were determined according to the following ratio: (area of expansion + area of retraction)/(area of earlier image + area of later image) (Kim and Wu, 1991a). The motility index varied over time at a mean of 0.36 (Fig. 4 and Table 2) for the active lamellipodia in normal cultures (23/37, see above). The active ruffling was not present in the rest of lamellipodia $(14 / 37)$,

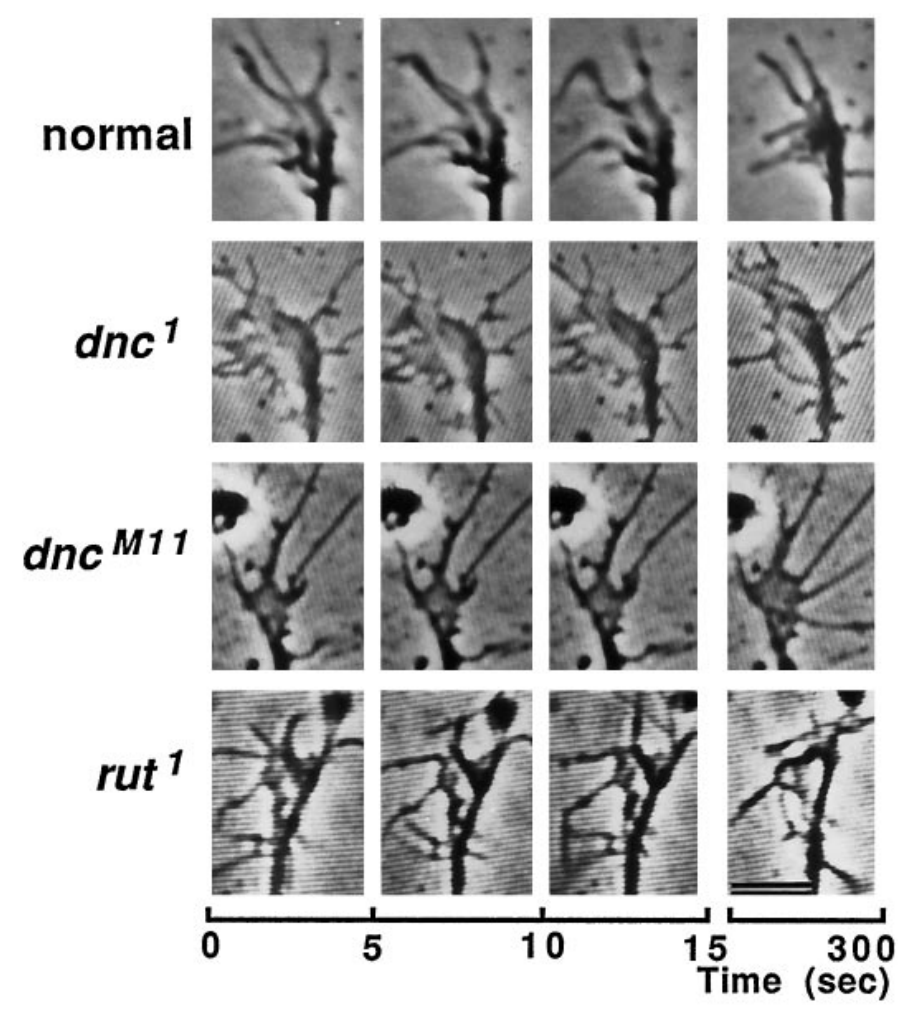

Figure 2. Time-lapse video micrographs of growth cones from normal, $d n c^{1}, d n c^{M 11}$, and $r u t^{1}$ neurons. Video-enhanced phase-contrast images of growth cones were photographed from the video monitor screen. The first three frames for each growth cone were captured at $5 \mathrm{sec}$ intervals and the last frame at $5 \mathrm{~min}$ after the first frame. Note the active lamellipodial ruffling and filopodial sweeping in the normal growth cone (top). Growth cones of rut ${ }^{1}$ and two alleles of $d n c$ showed greatly reduced motility (bottom three rows). Scale bar, $5 \mu \mathrm{m}$. 


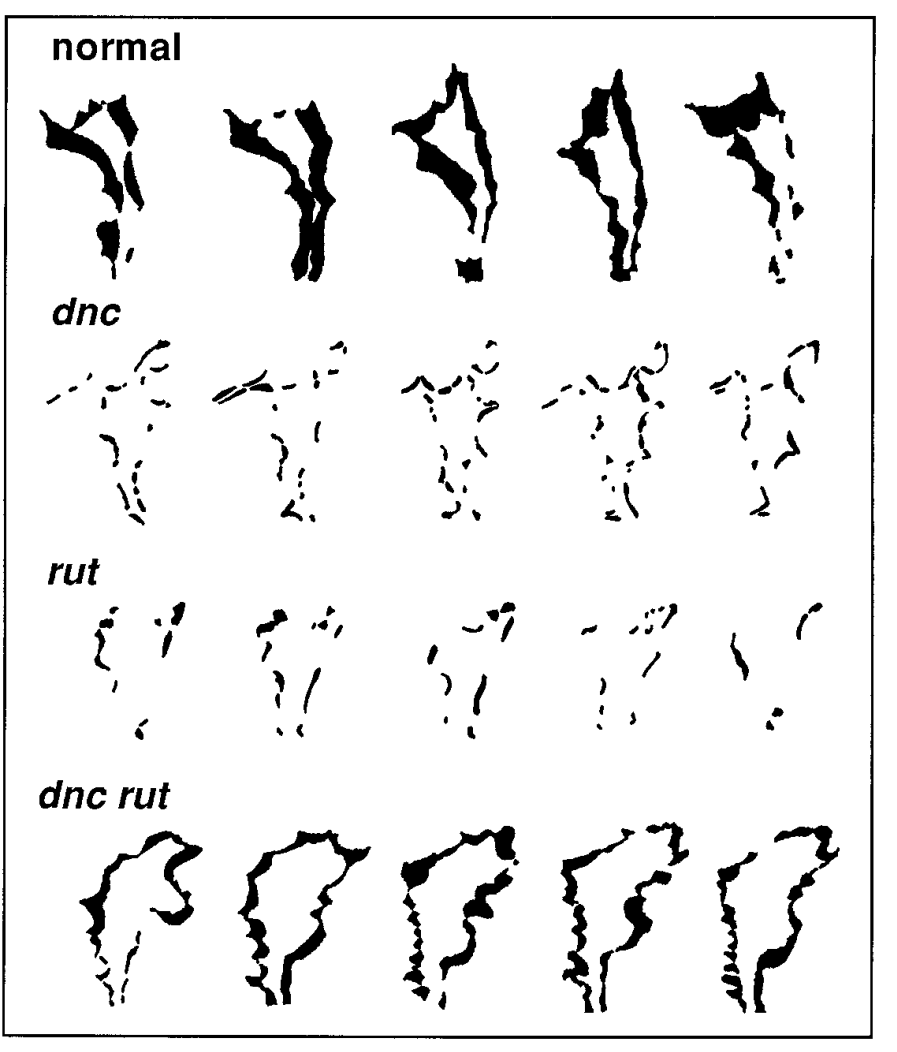

Figure 3. Difference pictures of growth cones of normal, dnc, rut, and double-mutant neurons. Areas in black, which represent lamellipodial expansion and retraction, were generated by superimposing two consecutive frames of a growth cone lamellipodium $5 \mathrm{sec}$ apart. Note the reduced motility in the single mutants and the larger displacement of lamellipodia seen in both normal and $d n c$ rut double-mutant growth cones.

which expanded and retracted slowly, giving a motility index of $\sim 0.10$ or less (data not shown; also see Kim and Wu, 1991a).

In contrast, throughout this study, we never found actively ruffling lamellipodia (558 cells in 24 cultures) in $d n c$ or rut cultures, but observed only slow expansion and retraction (Fig. 3) with a motility index comparable to the that of inactive lamellipodia in normal cultures. The average motility index of lamellipodia was $0.10,0.09,0.07$, and 0.07 for $d n c^{1}, d n c^{M 11}, d n c^{M 14}$, and $r u t^{l}$, respectively (Fig. 4 and Table 2). Figure 4 shows examples of the time course for the motility index in growth cones from normal, $d n c^{M 14}$, and $r u t^{1}$. Motility indices were obtained during a period of 12-13 successive $5 \mathrm{sec}$ intervals and from several later frames collected at the same intervals for up to $12 \mathrm{~min}$. The defects observed in the mutant strains are most likely attributable to the $d n c$ and rut loci, because consistent phenotypes were seen in different independent isolates.

\section{Effects of db-cAMP and forskolin on normal growth cones}

Because $d n c$ and rut affect the enzymes that regulate cAMP metabolism, it is of interest to determine whether acute application of pharmacological agents that perturb cAMP regulation in normal neurons would mimic the $d n c$ and rut phenotype. The membrane-permeable cAMP analog db-cAMP and the drug forskolin, which stimulates $\mathrm{AC}$, have been used widely in perfusion experiments. We found that motile lamellipodia in normal Drosophila neurons were very sensitive to db-cAMP. Figure 5 shows a series of time-lapse images observed during db-cAMP perfusion.
The growth cone displayed high motility before the onset of perfusion (Fig. 5a,b) and was not affected by perfusion of normal medium (up to $200 \mu \mathrm{l} / \mathrm{min}$ in laminar flow; Fig. $5 c, d$ ). Within 2 min of $25 \mu \mathrm{M} \mathrm{db}$-cAMP perfusion, the rapid lamellipodial movement declined gradually (Fig. 5e,f). Even though the morphology of the thin lamellipodia remained intact in the presence of $25 \mu \mathrm{M}$ db-cAMP, a brief ( $4 \mathrm{~min}$ ) exposure had a long-lasting effect on motility (Fig. 5g,h). In fact, high level motility did not recover within $2 \mathrm{hr}$ after returning to normal medium. Only slow extension and retraction of lamellipodia (comparable to $d n c$ growth cones shown in Fig. 2) were observed. Even $10 \mu \mathrm{M} \mathrm{db}$-cAMP affects lamellipodial motility, and only partial recovery was obtained after a brief exposure of 4 min (data not shown). In contrast, a higher concentration of db-cAMP $(50 \mu \mathrm{M})$ not only rapidly inhibited lamellipodial motility but also transformed growth cones into thick, club-shaped endings within 6 min (Fig. 5i,j).

We also found that $50 \mu \mathrm{M}$ forskolin had an effect similar to 25 $\mu \mathrm{M} \mathrm{db}$-cAMP (Fig. 6). A series of time-lapse images of a growth cone from a forskolin perfusion experiment in normal culture is shown in Figure 6. A high level of growth cone motility was maintained during a perfusion with standard medium (Fig. 6a,b). Shortly after switching to a medium containing $50 \mu \mathrm{M}$ forskolin, however, lamellipodial movement decreased gradually and filopodia retracted. After 2 min of perfusion, lamellipodial ruffling was inhibited (Fig. $6 c, d$ ). Minor changes in lamellipodial shape could be detected only over longer time periods.

The motility was still suppressed long after reperfusion with normal medium (Fig. $6 e, f$ ). After 4 min of wash, slow expansion of lamellipodia was barely detectable (Fig. $6 f$ ); however, lamellipodial movement was not restored to normal level during a $2 \mathrm{hr}$ observation period on returning to normal medium (not shown). Throughout this study, consistent arrest of motility by both dbcAMP and forskolin was observed in 33 growth cones from 25 independent cultures.

\section{Effects of db-cAMP on rut growth cones}

The above results indicate that abnormally high cAMP levels could lead to the arrest of growth cone motility; however, the observed retardation of growth cone motility in rut neurons (Figs. 2-4) suggests that an abnormally low level of cAMP synthesis could also cause a similar effect. To test whether restoration of an optimal level of cAMP could bring about normal growth cone motility, we applied db-cAMP and forskolin to rut growth cones in culture at the time of plating. Indeed, motility of growth cones was rescued, and clear lamellipodial movement could be observed in rut cultures chronically incubated with 1.5-2.5 $\mu \mathrm{M}$ db-cAMP for 40-56 hr. Twenty-two of 50 growth cones subjected to continuous observation for 2-10 min in 10 independent rut cultures showed active lamellipodial movement, which was readily detectable in 10 sec intervals (Fig. 7a-d). Ruffling movements of lamellipodia and extension and retraction of filopodia were evident. Most importantly, the recovery of growth cone motility assisted by db-cAMP in rut cultures seemed to require an optimal range of concentrations. When rut neurons were incubated with $5 \mu \mathrm{M}$ or higher concentrations of db-cAMP, no clear recovery in motility was observed. Instead, growth cones showed phase-dark images. Thus, too high concentrations of db-cAMP when applied chronically to rut growth cones resulted in phenotypes resembling the effect of forskolin or high concentrations of db-cAMP on normal growth cones.

Another set of experiments used perfusion of db-cAMP to determine the response time of rut growth cones. Significantly, 


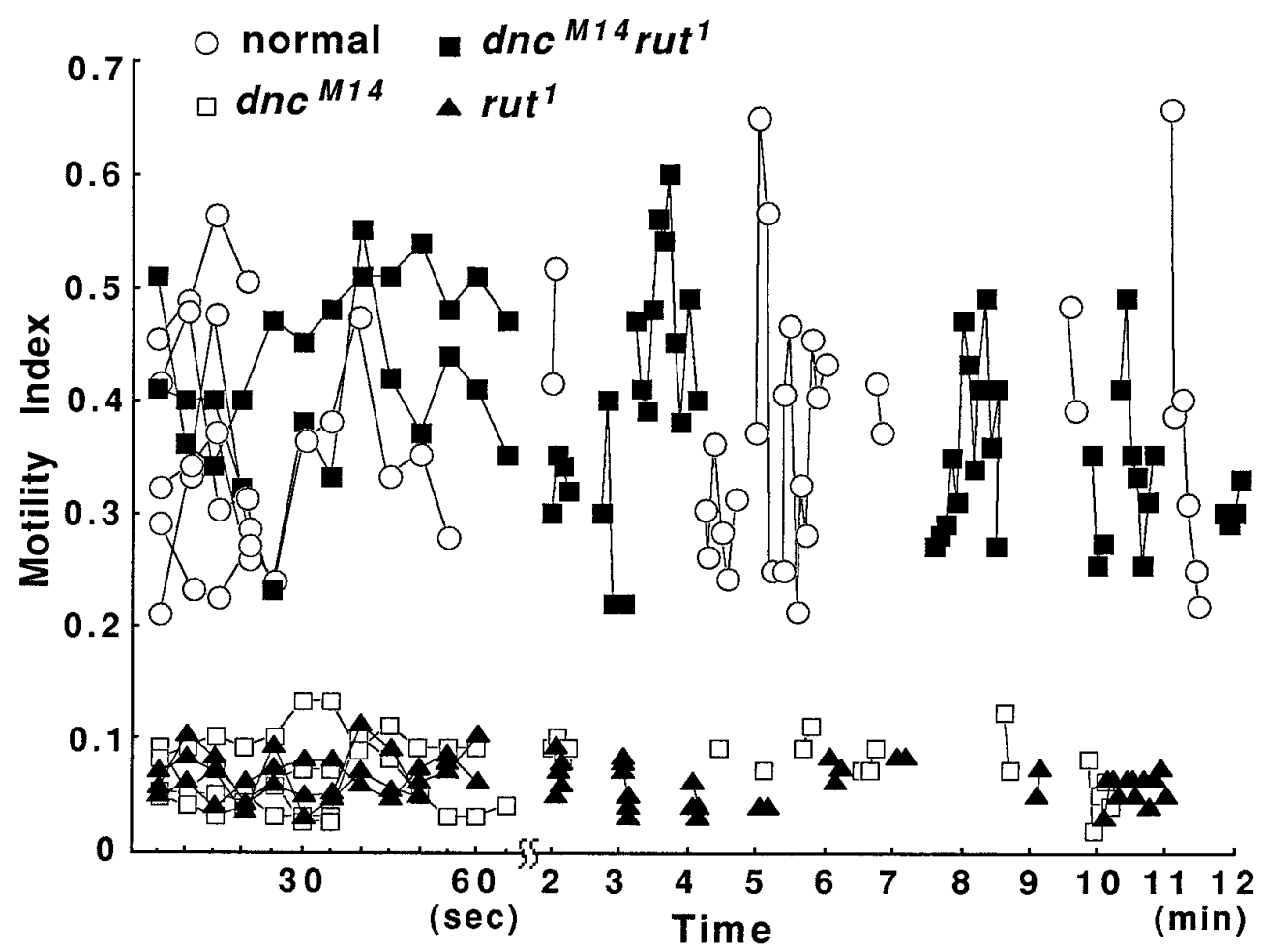

Figure 4. Motility index in normal, dnc, rut, and double-mutant growth cones plotted over time. The motility index was determined on the basis of consecutive difference pictures at $5 \mathrm{sec}$ intervals (see Fig. 3). For each genotype, the sample included a total of 57-115 difference pictures based on three to five growth cones. Data points for individual growth cones are connected. The single mutants had similar motility indices, which were significantly lower than those of normal and double-mutant neurons $(p<0.001$, Student's $t$ test). Segments of two different time scales are presented to show the short-term and long-term changes.

immobile rut lamellipodia responded rapidly to acute perfusion of db-cAMP-containing medium $(\sim 5.0-12.5 \mu \mathrm{M})$. Visible lamellipodial expansion began within 1 min after the onset of db-cAMP perfusion (Fig. $7 g, h$ ). The response reached the plateau within 2-3 min, showing lamellipodial ruffling and filopodial sweeping (Fig. $7 i, j)$. On returning to normal perfusion medium, the growth cone activity subsided within $1 \mathrm{~min}$ and gradually retracted to the original immobile state (Fig. $7 k, l$ ).

In contrast to the results of db-cAMP, forskolin at concentrations between 5 and $12.5 \mu \mathrm{M}$ never brought about active growth cone motility in rut cultures in chronic incubation or acute perfusion experiments. This is consistent with the previous observation that $\mathrm{AC}$ in rut adult homogenates fails to respond to forskolin stimulation (Dudai et al., 1985). Growth cones in rut cultures incubated with forskolin were typically phase-dark and appeared thicker at lamellipodial edges.

\section{Counterbalancing effects of $d n c$ and rut on growth cone motility}

The $d n c$ gene codes for cAMP PDE, which hydrolyzes cAMP, whereas the rut locus codes for a $\mathrm{Ca}^{2+} /$ calmodulin-sensitive $\mathrm{AC}$ that synthesizes cAMP. Even though the $d n c$ and rut genes have an opposite effect on cAMP metabolism, as we described above, mutations of either gene result in greatly retarded motility of growth cone. With these data, it is desirable to obtain an independent line of evidence showing that an optimum level of cAMP
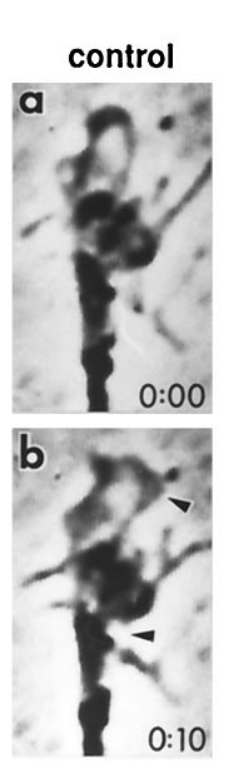
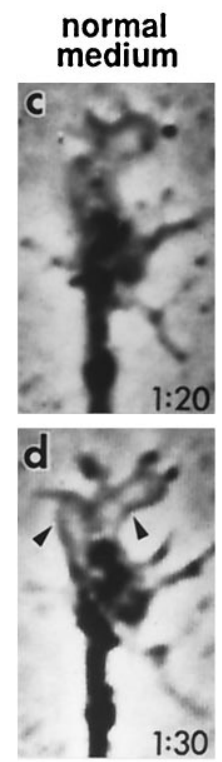
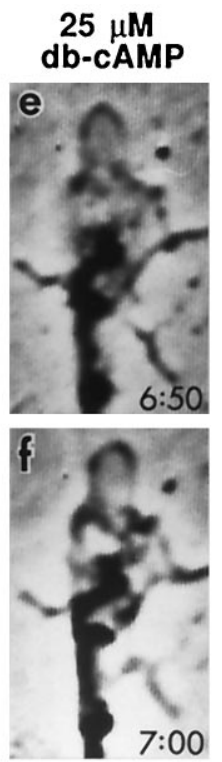
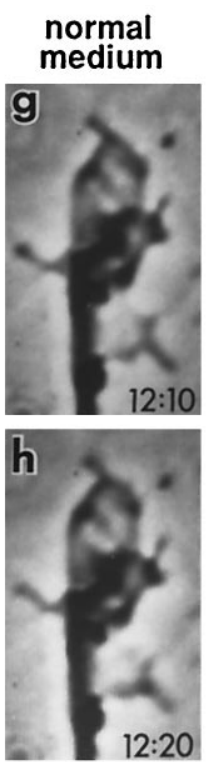
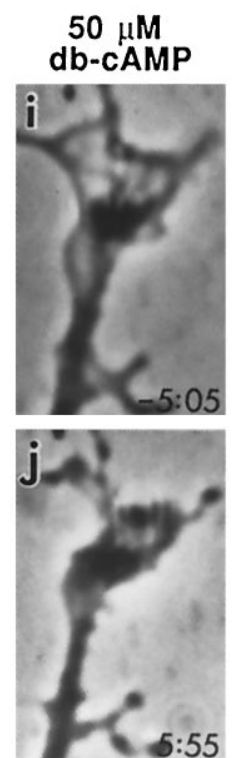

Figure 5. Arrest of growth cone motility in normal neurons by perfusion with db-cAMP. The growth cone of a normal neuron displayed high motility (arrowheads) before the onset of perfusion $(a, b)$ and was not affected (arrowheads) by perfusion of normal medium $(c, d)$. Within 2 min of perfusion with $25 \mu \mathrm{M} \mathrm{db}$-cAMP, growth cone motility was severely retarded $(e, f)$. Long after returning to normal medium, the arrest was not reversed $(g, h$; see text). Shown for a different growth cone $(i, j)$, a higher concentration of dbcAMP $(50 \mu \mathrm{M})$ caused not only motility arrest but also retraction of lamellipodia. For $a-h$, the onset of the first normal medium, $25 \mu \mathrm{M}$ db-cAMP, and second normal medium perfusion was at $30 \mathrm{sec}$, $4.5 \mathrm{~min}$, and $8.5 \mathrm{~min}$, respectively. For $i$ and $j, 50$ $\mu \mathrm{M} \mathrm{db}$-cAMP perfusion started at time 0 . 


\section{normal medium}
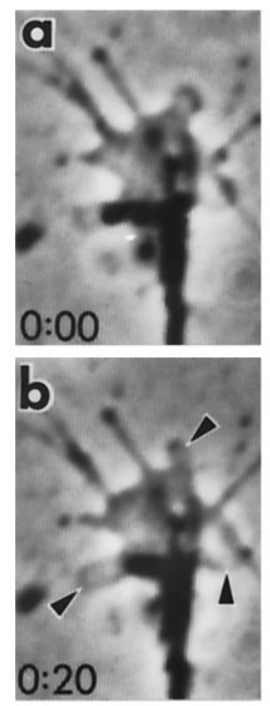

$50 \mu \mathrm{M}$ forskolin
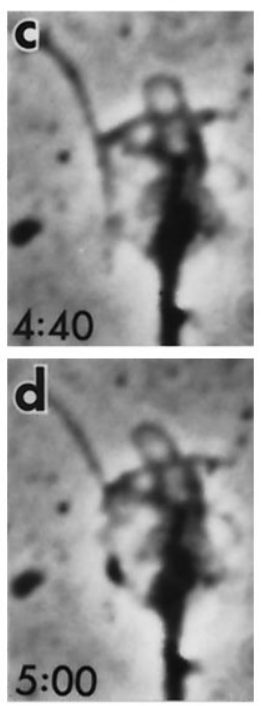

normal medium

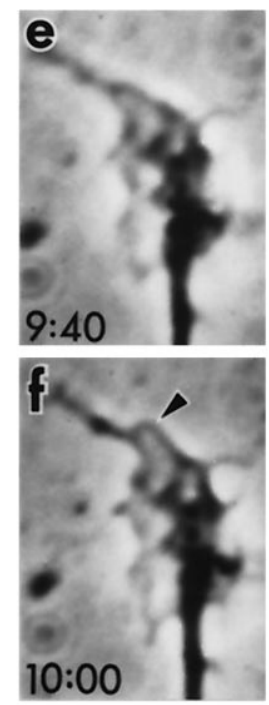

Figure 6. Reversible arrest of growth cone motility in normal neuron by forskolin. Active ruffling of lamellipodia (arrowheads) during normal medium perfusion $(a, b)$ was arrested soon after perfusion with medium containing $50 \mu \mathrm{M}$ forskolin, a drug stimulating $\operatorname{AC}(c, d)$. Lowered growth cone motility by forskolin is similar to that seen in $d n c$ neurons (see Fig. 2 ). Motility resumed (arrowhead) after wash by perfusion with normal medium $(e, f)$. The onset of the first normal medium, $50 \mu \mathrm{M}$ forskolin, and second normal medium was at $-2 \mathrm{~min}, 2 \mathrm{~min}$, and $6 \mathrm{~min}$, respectively.

is required to maintain normal growth cone motility. One key experiment, made possible by the use of genetics, was to pair the two mutations $d n c$ and rut in a natural setting of double-mutant neurons and to look for compensation for the effect of abnormal cAMP levels caused by each single mutation.

We examined the dnc rut double-mutant growth cones in culture. The morphology of $d n c$ rut growth cones was almost the same as normal for several morphometric parameters, including number and length of filopodia and area of lamellipodia (Table 2). Interestingly, the motility of $d n c$ rut growth cones was active enough to be detected in time-lapse observations with $5 \mathrm{sec}$ intervals (Fig. 8, bottom). The recovery of motility by the counterbalancing effects of $d n c$ and rut was clearly evident in difference pictures (Fig. 3, bottom) and the motility index over time (Fig. 4). The motility index of the double mutant was $0.36+0.08$, which appears identical to that of normal cultures $(0.36+0.09$; see Table 2).

\section{DISCUSSION}

\section{Growth cones in learning mutants}

This is the first demonstration of altered growth cone properties in learning and memory mutants. We exploited a larval CNS culture system (Wu et al., 1983; Kim and Wu, 1987, 1991a; Wu, 1988) that allows for direct observations of growth cones in isolated $d n c$ and rut neurons. Motility and morphology of the growth cone were examined in monopolar type III neurons in which their larger sizes readily allow contrast-enhancement video recording. We found in mutant neurons striking retardation in the motility of phase-light growth cone lamellipodia, which have been shown to be highly motile in normal cultures (Kim and $\mathrm{Wu}$, 1991a).
In addition, $d n c$ and rut mutations also led to a darker appearance of phase-dark lamellipodia, which generally show little motility and are thought to be associated with neurons of older ages in normal cultures (Kim and $\mathrm{Wu}, 1991 \mathrm{a}$ ). The same is true for other culture systems that growth cones in older neurons show darker phase images and reduced movement in time-lapse studies (Argiro et al., 1984; Hadley et al., 1985; Goldberg and Burmeister, 1986; Goldberg et al., 1991). Nevertheless, the reduced lamellipodial motility of $d n c$ and rut neurons is not associated with accelerated aging in neurons, because the ratio between phaselight and phase-dark growth cones did not differ between normal and mutant cultures (Table 1) and no active lamellipodia were seen in younger cultures of $<8 \mathrm{hr}$ of age (Y.-T. Kim, P. Taft, and C.-F. Wu, unpublished data). In contrast, high motility still persisted in some lamellipodia in normal cultures as old as $3 \mathrm{~d}$.

Reduced lamellipodial motility in $d n c$ and rut neurons was not correlated directly to rate of neurite extension and neuronal growth. Although the growth rate of mutant neurons has not been determined using time-lapse recording, sampling of neuritic length in a large number of cultures of different ages indicated that the neurite extension rate (Kim and $\mathrm{Wu}, 1987$ ) was not retarded in $d n c$ and rut cultures (P. Taft and C.-F. Wu, unpublished data). The lamellipodial activity could thus be considered to reflect the exploratory and searching behavior of growth cones, which is not directly correlated to neurite elongation (Kim and $\mathrm{Wu}, 1991 \mathrm{a})$ but may influence the directionality of outgrowth and the proper connectivity in developing neurons or activitydependent modification of mature neurons in the nervous system (Baier and Bonhoeffer, 1991; Marcus et al., 1995). Defects leading to reduced exploratory activity of growth cones in mutant cultures thus do not necessarily decrease nerve branching and elongation in vivo, as indicated by the previously reported overgrowth and sprouting of motor axons in the larval neuromuscular junction of dnc mutants (Zhong et al., 1992).

Modulation of synaptic efficacy as the physiological basis for learning has long been subjected to intensive investigation in several preparations (Kandel and Schwartz, 1982; Byrne and Kandel, 1996). Physiological studies in Drosophila have also shown that activity-dependent short-term plasticity in synaptic transmission is altered severely at larval neuromuscular junctions in learning mutants, including $d n c$ and rut (Zhong and Wu, 1991; Wang et al., 1994). In contrast, growth regulation of nerve terminals as morphological correlates of learning has been characterized extensively only in more recent years (Bailey and Kandel, 1993).

Our findings show that the cellular machinery regulating growth cone behavior is demonstrably different in cultures of $d n c$ and rut neurons. Defects in the same cellular machinery may influence neuronal development in vivo and contribute to the altered morphological plasticity observed previously in Drosophila. Both adult $d n c$ and rut flies are known to have fewer fiber numbers in the mushroom bodies, which are involved in olfactory learning (Balling et al., 1987), and increased terminal varicosities of an identified sensory neuron (Corfas and Dudai, 1991). In addition, the terminals of larval motor neurons have been shown to increase the number of branching and varicosities in $d n c$ larvae, which can be suppressed by the rut mutation in dnc rut double mutants (Zhong et al., 1992).

\section{Optimal levels of cAMP in growth cone regulation}

Our findings suggest that normal lamellipodial motility in developing Drosophila neurons requires optimal cAMP levels with an 


\section{Incubation}

\section{db-cAMP, $2.5 \mu \mathrm{M}$}
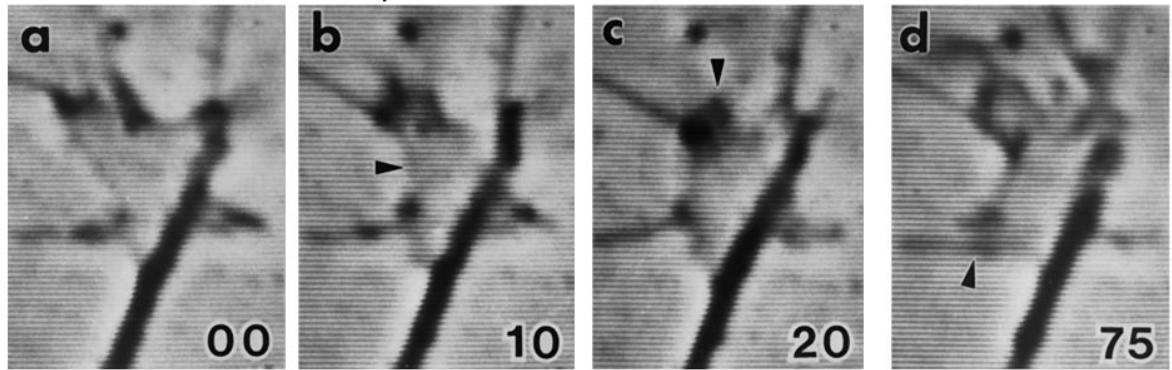

Perfusion

\section{normal medium}
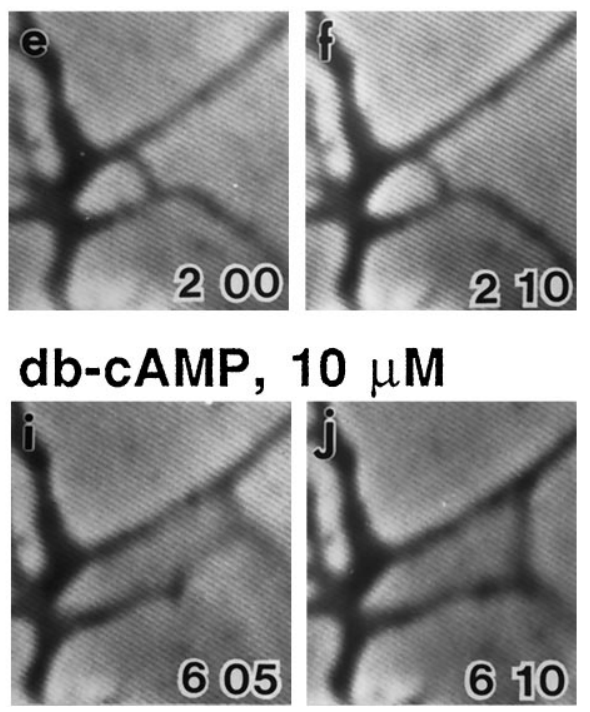

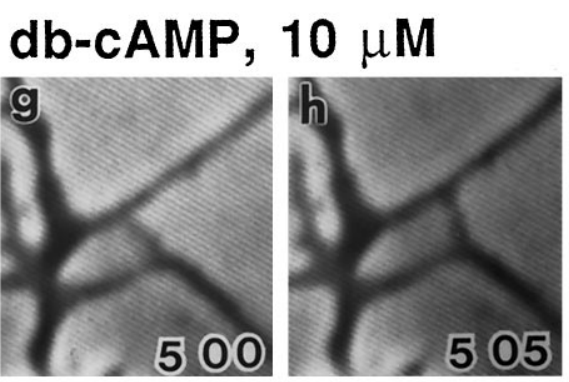

\section{normal medium}

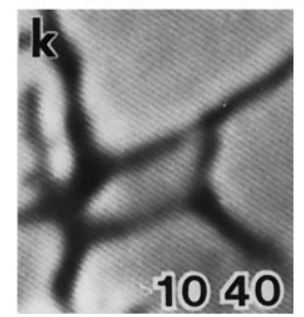

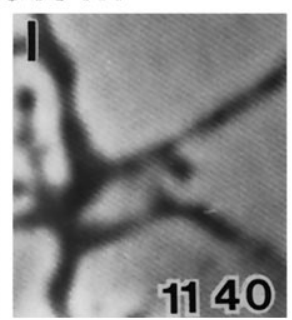

Figure 7. Rescue of growth cone motility in rut neurons by db-cAMP. Growth cone motility in the rut neuron was restored, and clear lamellipodial movement could be observed when incubated with $2.5 \mu \mathrm{M} \mathrm{db}$-cAMP from the time of plating for $24 \mathrm{hr}$. Ruffling movement of lamellipodia (arrowheads) was readily detectable at $10 \mathrm{sec}$ intervals $(a-c)$, and considerable changes in growth cone shape were seen after a longer period $(d, 75 \mathrm{sec})$. $e-l$ show the perfusion experiment for another growth cone. The rut growth cone did not respond to perfusion with normal medium $(e, f)$. Within 1 min of $10 \mu \mathrm{M} \mathrm{db}$-cAMP perfusion, rut growth cone became motile with rapid lamellipodial expansion $(g, h)$ and filopodial sweeping $(i, j)$. Motility subsided and the growth cone returned to the original state after the wash with normal medium perfusion $(k, l)$. operational range of dynamic control, as summarized below. First, either increased or reduced cAMP beyond a normal range in $d n c$ and rut (Byers et al., 1981; Livingstone et al., 1984) leads to arrest of lamellipodial motility (Figs. 2-4). Second, normal neurons subjected to perfusion with medium containing forskolin or high concentrations of db-cAMP phenocopy the $d n c$ defects (Figs. 5, 6). Third, lamellipodial motility in rut neurons could be rescued when incubated with relatively low, but not high, concentrations of db-cAMP or when perfused with intermediate levels of dbcAMP (Fig. 7). Finally, in double mutants, in which cAMP levels are close to normal (Livingstone et al., 1984), lamellipodial activity was restored (Fig. 8). The ability to manipulate growth cone motility by mutations and pharmacological treatment affecting synthesis, degradation, or the general levels of cAMP support a specific role of cAMP in regulating lamellipodia expansion or retraction. The bell-shaped dependence on cAMP levels corroborates the recently emerging theme found in other systems that many cellular regulatory processes are achieved by small dynamic local modifications around a set point of a cellular messenger.

The exact mechanisms responsible for the restoration of growth cone motility in the double mutants await further studies: notably, certain phenotypes could be restored completely or partially, whereas others could be worsened in the double mutants. It is worth noting that even though the direction of concentration change in cAMP is opposite in the two mutants, $d n c$ and rut growth cones display similar behavioral abnormalities, their neurons show similar aberrant excitability patterns (Zhao and $\mathrm{Wu}$, 1994), and adult flies are similarly deficient in learning tasks (Tully and Quinn, 1985; Davis, 1996). Counterbalancing effects in the double mutants is seen in intracellular cAMP levels (Livingstone et al., 1984), growth cone motility (this work), and nerve terminal arborization (Zhong et al., 1992). Nevertheless, when $d n c$ and rut were recombined in double mutants, more severe phenotype was seen in learning deficiency (Livingstone et al., 1984), physiological defects of habituation in an identified escape circuit (Engel and $\mathrm{Wu}, 1994,1996)$, and aberrant excitability of cultured neurons (Zhao and $\mathrm{Wu}, 1994)$.

Explanations for the apparent complexity in the actions of and interactions between rut and $d n c$ may include several well established cellular mechanisms. It is known that many enzymes are compartmentalized and colocalized with downstream targets to mediate local physiological events. Some functional consequences of cAMP regulation may depend on its dynamic changes rather than its steady-state levels. Furthermore, compensatory interactions among second messenger systems may increase the complexity in the outcome of the cAMP regulation.

Several entry points to initiating studies of these problems have been suggested by previous observations. Rearrangement of actin filaments and microtubules has been implicated in the dynamics of lamellipodia and filopodia (Bray, 1991; Lin et al., 1994). In 


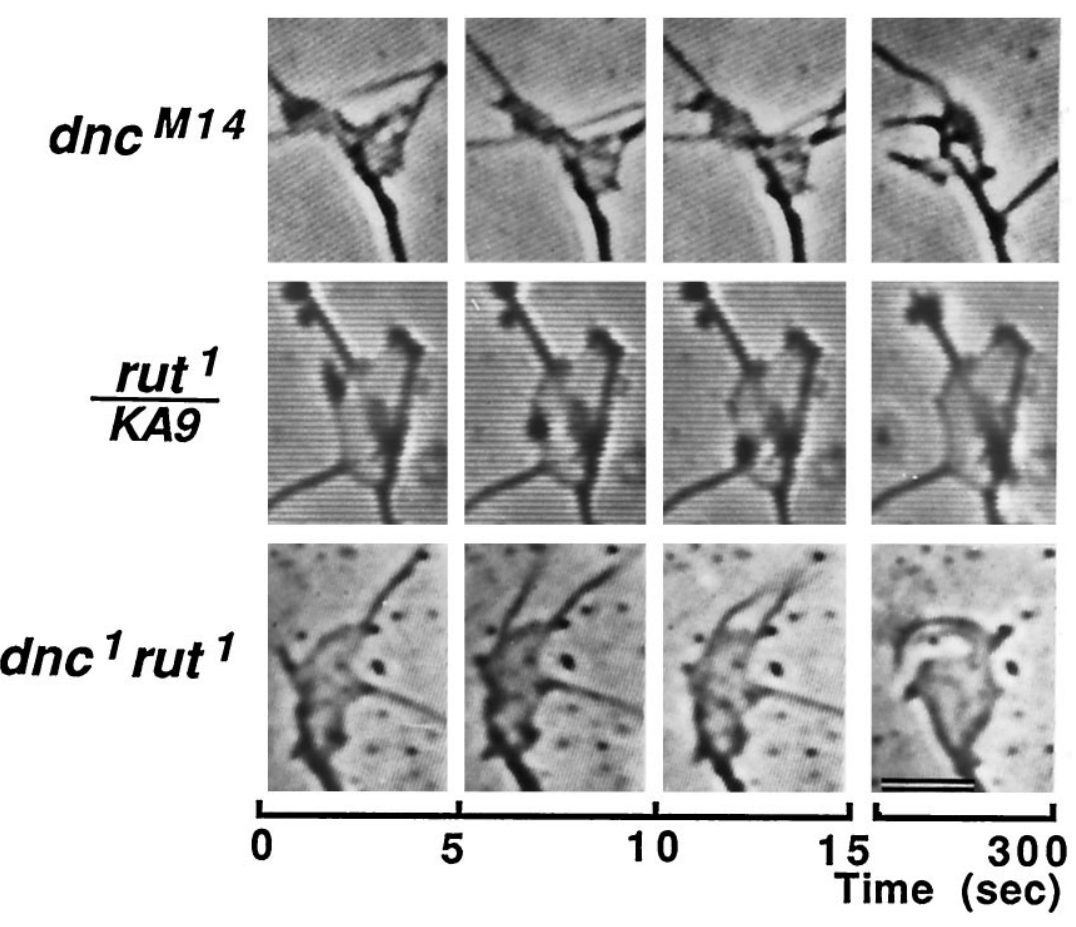

Figure 8. Time-lapse video micrographs of $d n c^{M 14}$, $r u t^{1} / K A 9$, and $d n c^{1} r^{r} t^{1}$ growth cones. Growth cones were photographed and displayed in the same way as in Figure 2. Note that active lamellipodial ruffling and filopodial sweeping were restored in the $d n c^{1} r u t^{1}$ double-mutant growth cone (bottom). Growth cones of both $d n c^{M 14}$ and $r u t^{1} / K A 9$ (heterozygote of $r u t^{1}$ over $K A 9$, a chromosome bearing a deficiency of the rut locus) showed greatly reduced motility (upper two rows). Same magnification as in Figure 2.

\author{
$d n c^{1}$ rut $^{1}$
}

mulation of these enzymes in the mushroom bodies (Han et al., 1992; Nighorn et al., 1994).

In other invertebrate systems, including Aplysia, the role of cAMP in several simple forms of learning has been well established (Byrne and Kandel, 1996). It is thought that cAMP contributes to the initiation of short-term memory by protein phosphorylation and to the consolidation of long-term memory by mechanisms involving gene expression (Goelet et al., 1986; Byrne and Kandel, 1996). Isolation of the Drosophila mutants $d n c$ and rut with defects in cAMP metabolism allows identification of phenotypes at the cellular level (Zhong and Wu, 1991; Zhong et al., 1992; Engel and Wu, 1994, 1996; Zhao and Wu, 1994; this work) and provides independent evidence for the critical role of cAMP in neuronal plasticity underlying learning.

It will be of importance to determine whether the altered growth cone properties observed in $d n c$ and rut cultures could be correlated to abnormal exploring behavior and growth pattern of developing neurons in vivo. Preparations for direct time-lapse observations in the developing Drosophila embryo exist in which growth cone motility and neurite growth may be observed continuously in situ (Halpern et al., 1991; Keshishian et al., 1993).

The current culture system allows the first analysis of the defects in $d n c$ and rut growth cones. Further development of this analysis may exploit mutations of additional genes and include phenomena related to interactions among different cell populations. High-density cultures can be initiated to study interactions among neurons of different genotypes or the process of synapse formation between different neuronal types, which may be vitally marked by using the green fluorescent protein reporter gene (Yeh et al., 1995). Modifications of our experimental system (Wu et al., 1990; Zhao et al., 1995) may allow determination of how growth cone motility is regulated at the local level. For instance, dbcAMP and related pharmacological agents can be applied locally to the various subcellular regions via a micropipette (Lohof et al., 1992; Zheng et al., 1996). The results presented here provide the 
groundwork for additional studies of the roles of second messenger systems and regulation of growth cone properties that are important in neuronal development and neural plasticity.

\section{REFERENCES}

Aceves-Pina EO, Booker R, Duerr JS, Livingstone MS, Quinn WG, Smith RF, Sziber PP, Tempel BL, Tully TP (1983) Learning and memory in Drosophila, studied with mutants. Cold Spring Harb Symp Quant Biol 48:831-840.

Adam G, Friedrich P (1988) Microtubule-associated cyclic AMPdependent protein kinase in Drosophila melanogaster. J Neurochem 51:1014-1022.

Argiro V, Bunge MB, Johnson MI (1984) Correlation between growth cone form and movement and their dependence on neuronal age. J Neurosci 4:3051-3062.

Baier H, Bonhoeffer F (1991) Axon guidance in vitro by a target-derived cell membrane component. In: The nerve growth cone (Letourneau PC, Kater SB, Macagno ER, eds), pp 195-206. New York: Raven.

Bailey C, Kandel ER (1993) Structural changes accompanying memory storage. Annu Rev Physiol 55:397-426.

Balling A, Technau GM, Heisenberg M (1987) Are the structural changes in adult Drosophila mushroom bodies memory traces? Studies on biochemical learning mutants. J Neurogenet 4:65-73.

Bray D (1991) Cytoskeletal basis of nerve axon growth. In: The nerve growth cone (Letourneau PC, Kater SB, Macagno ER, eds), pp 7-17. New York: Raven.

Bray D, Chapman K (1985) Analysis of microspike movements of the neuronal growth cone. J Neurosci 5:3204-3213.

Byers D, Davis RL, Kiger JA (1981) Defect in cyclic AMP phosphodiesterase due to the dunce mutation of learning in Drosophila melanogaster. Nature 289:79-81.

Byrne JH, Kandel ER (1996) Presynaptic facilitation revisited: state and time dependence. J Neurosci 16:425-435.

Chen CN, Denome S, Davis RL (1986) Molecular analysis of cDNA clones and the corresponding genomic coding sequences of the Drosophila dunce ${ }^{+}$gene, the structural gene for cAMP phosphodiesterase. Proc Natl Acad Sci USA 83:9313-9317.

Corfas G, Dudai Y (1990) Adaptation and fatigue of a mechanosensory neuron in wild-type Drosophila and in memory mutants. J Neurosci 10:491-499.

Corfas G, Dudai Y (1991) Morphology of a sensory neuron in Drosophila is abnormal in memory mutants and changes during aging. Proc Natl Acad Sci USA 88:7252-7256.

Davis RL (1996) Physiology and biochemistry of Drosophila learning mutants. Physiol Rev 76:299-317.

Davis RL, Kiger JA (1981) dunce mutants of Drosophila melanogaster: mutants defective in the cyclic AMP phosphodiesterase enzyme system. J Cell Biol 90:101-107.

Dudai Y, Jan Y-N, Byers D, Quinn WG, Benzer S (1976) dunce, a mutant of Drosophila deficient in learning. Proc Natl Acad Sci USA 73:1684-1688.

Dudai Y, Uzzan A, Zvi S (1983) Abnormal activity of adenylate cyclase in the Drosophila memory mutant rutabaga. Neurosci Lett 42:207-212.

Dudai Y, Sher B, Segal D, Yovell Y (1985) Defective responsiveness of adenylate cyclase to forskolin in the Drosophila memory mutant rutabaga. J Neurogenet 2:365-380.

Engel JE, Wu C-F (1994) Repetitive conditioning of the Drosophila giant fiber response is altered in a mutant with learning deficiencies. Soc Neurosci Abstr 20:803.

Engel JE, Wu C-F (1996) Altered habituation of an identified escape circuit in Drosophila memory mutants. J Neurosci 16:3488-3499.

Forscher P, Kaczmarek LK, Buchanan J, Smith SJ (1987) Cyclic AMP induces changes in distribution and transport of organelles within growth cones of Aplysia bag cell neurons. J Neurosci 7:3600-3611.

Frey U, Huang Y-Y, Kandel ER (1993) Effects of cAMP simulate a late stage of LTP in hippocampal CA1 neurons. Science 260:1661-1664.

Goelet P, Castellucci VF, Schacher S, Kandel ER (1986) The long and the short of long-term memory: a molecular framework. Nature 322:419-422.

Goldberg DJ, Burmeister D (1986) Stages in axon formation: observations of growth of Aplysia axons in culture using video-enhanced contrast-differential interference contrast microscopy. J Cell Biol 103:1921-1931.

Goldberg DJ, Burmeister D, Rivas RJ (1991) Video microscopic analysis of events in the growth cone underlying axon growth and the regulation of these events by substrate-bound proteins. In: The nerve growth cone (Letourneau PC, Kater SB, Macagno ER, eds), pp 79-95. New York: Raven.

Hadley RD, Bodnar DA, Kater SB (1985) Formation of electrical synapses between isolated, cultured Helisoma neurons requires mutual neurite elongation. J Neurosci 5:3145-3153.

Halpern ME, Chiba A, Johansen J, Keshishian H (1991) Growth cone behavior underlying the development of stereotypic synaptic connections in Drosophila embryos. J Neurosci 11:3227-3238.

Han P-L, Levin LR, Reed RR, Davis RL (1992) Preferential expression of the Drosophila rutabaga gene in mushroom bodies, neural centers for learning in insects. Neuron 9:619-627.

Heisenberg M, Borst A, Wagner S, Byers D (1985) Drosophila mushroom body mutants are deficient in olfactory learning. J Neurogenet 2:1-30.

Heisenberg M, Heusipp M, Wanke C (1995) Structural plasticity in the Drosophila brain. J Neurosci 15:1951-1960.

Kandel ER, Schwartz JH (1982) Molecular biology of learning: modulation of transmitter release. Science 218:433-443.

Keshishian H, Chiba A, Chang TN, Halfon M, Harkins EW, Jarecki J, Wang LS, Anderson MD, Cash S, Halpern ME, Johansen J (1993) Cellular mechanisms governing synaptic development in Drosophila melanogaster. J Neurobiol 24:757-787.

Kim Y-T, Wu C-F (1987) Reversible blockage of neurite development and growth cone formation in neuronal cultures of a temperaturesensitive mutant of Drosophila. J Neurosci 7:3245-3255.

Kim Y-T, Wu C-F (1991a) Distinctions in growth cone morphology and motility between monopolar and multipolar neurons in Drosophila CNS culture. J Neurobiol 22:263-275.

Kim Y-T, Wu C-F (1991b) Effects of cAMP on growth cone motility in cultured CNS neurons: analysis of Drosophila memory mutants, dunce and rutabaga. Soc Neurosci Abstr 17:1056.

Kim Y-T, Taft P, Wu C-F (1995) Growth cone morphology and behavior in cultured CNS neurons of Drosophila memory mutants. Soc Neurosci Abstr 21:169.

Levin LR, Han P-L, Hwang PM, Feinstein PG, Davis RL, Read RR (1992) The Drosophila learning and memory gene rutabaga encodes a $\mathrm{Ca}^{2+}$ /calmodulin-responsive adenylyl cyclase. Cell 68:479-489.

Lin C-H, Thompson CA, Forscher P (1994) Cytoskeletal reorganization underlying growth cone motility. Curr Opin Neurobiol 4:640-647.

Livingstone MS, Sziber PP, Quinn WG (1984) Loss of calcium/calmodulin responsiveness in adenylate cyclase of rutabaga, a Drosophila learning mutant. Cell 37:205-215.

Lohof AM, Quillan M, Dan Y, Poo M-m (1992) Asymmetric modulation of cytosolic cAMP activity induces growth cone turning. J Neurosci 12:1253-1261.

Marcus RC, Blazeski R, Godement P, Mason CA (1995) Retinal axon divergence in the optic chiasm: uncrossed axons diverge from crossed axons within a midline glial specialization. J Neurosci 15:3716-3729.

Mohler JD (1977) Developmental genetics of the Drosophila egg: identification of 59 sex-linked cistrons with maternal effects of embryonic development. Genetics 85:259-272.

Mons N, Cooper DMF (1995) Adenylate cyclases: critical foci in neuronal signaling. Trends Neurosci 18:536-542.

Nighorn A, Qiu Y, Davis RL (1994) Progress in understanding the Drosophila dnc locus. Comp Biochem Physiol 108B:1-9.

Qiu Y, Chen CN, Malone T, Richter L, Beckendorf SK, Davis RL (1991) Characterization of the memory gene dunce of Drosophila melanogaster. J Mol Biol 222:553-565.

Soll DR (1995) The use of computers in understanding how animal cells crawl. Int Rev Cytol 163:43-104.

Soll DR, Voss E, Varnum-Finnely B, Wessels D (1988) "Dynamic morphology system," a method for quantitating changes in shape, pseudopod formation, and motion in normal and mutant amoebae of Dictyostelium discoideum. J Cell Biochem 37:177-192.

Technau G (1984) Fiber number in the mushroom bodies of adult Drosophila melanogaster depends on age, sex and experience. J Neurogenet 1:113-126.

Tully T, Quinn WG (1985) Classical conditioning and retention in normal and mutant Drosophila melanogaster. J Comp Physiol [A] 157:263-277.

Vale RD (1987) Intracellular transport using microtubule based motors. Annu Rev Cell Biol 3:347-378. 
Wang J, Renger JJ, Griffith LC, Greenspan RJ, Wu C-F (1994) Concomitant alteration of physiological and developmental plasticity in Drosophila CaM kinase II-inhibited synapses. Neuron 13:1373-1384.

Wu C-F (1988) Neurogenetic studies of Drosophila central nervous system neurons in culture. In: Cell culture approaches to invertebrate neuroscience (Beadle DJ, Lees G, Kater SB, eds), pp 149-187. New York: Academic.

Wu C-F, Suzuki N, Poo M-m (1983) Dissociated neurons from normal and mutant Drosophila larval central nervous system in cell culture. J Neurosci 3:1888-1899.

Wu C-F, Sakai K, Saito M, Hotta Y (1990) Giant Drosophila neurons differentiated from cytokinesis-arrested embryonic neuroblasts. J Neurobiol 21:499-507.

Yeh E, Gustafson K, Boulianne G (1995) Green fluorescent protein as a vital marker and reporter of gene expression in Drosophila. Proc Natl Acad Sci USA 92:7036-7040.
Zhao M-L, Wu C-F (1994) Altered spike activity in cultured "giant" neurons derived from dunce and rutabaga mutants of Drosophila. Soc Neurosci Abstr 20:803.

Zhao M-L, Sable EO, Iverson LE, Wu C-F (1995) Functional expression of Shaker $\mathrm{K}^{+}$channels in cultured Drosophila "Giant" neurons derived from $S h$ cDNA transformants: distinct properties, distribution, and turnover. J Neurosci 15:1406-1418.

Zheng JQ, Wan J-j, Poo M-m (1996) Essential role of filopodia in chemotropic turning of nerve growth cone induced by a glutamate gradient. J Neurosci 16:1140-1149.

Zhong Y, Wu C-F (1991) Altered synaptic plasticity in Drosophila memory mutants with altered cAMP cascade. Science 251:198-201.

Zhong Y, Budnik V, Wu C-F (1992) Synaptic plasticity in Drosophila memory and hyperexcitability mutants: role of cAMP cascade. J Neurosci 12:644-651. 